\title{
Needs and Intentionality
}

\section{An Ontological Analysis and an Application to Public Services}

\author{
Luca BICCHERI ${ }^{\mathrm{a}}$, Roberta FERRARIO ${ }^{\mathrm{b}}$ and Daniele PORELLO ${ }^{\mathrm{b}}$ \\ ${ }^{\mathrm{a}}$ University of Urbino (Italy) \\ ${ }^{\mathrm{b}}$ ISTC-CNR, Laboratory for Applied Ontology, Trento (Italy)
}

\begin{abstract}
A thorough understanding of what needs are is fundamental for designing well-behaved information systems for many social applications and in particular for public services. Talking about needs pervades indeed the jargon of Public Administrations when motivating their service offering. In this paper, we propose an ontological analysis of needs, aiming at a principled disentangling of the different uses of the term. We leverage philosophical tradition on intentionality, for its rich understanding of mental entities, we compare it with the well-established BDI (Belief-Desire-Intention) tradition in knowledge representation, and we propose a formalisation of needs within the foundational ontology DOLCE. Throughout the paper, we motivate our analysis focusing on needs in public services.
\end{abstract}

Keywords. Need, satisfier, mental attitude, intentionality, intentional content, intentional object, public service, Public Administration.

\section{Introduction}

The mission of every Public Administration (PA) of a Welfare State is that of promoting the fulfillment of human rights and the well-being of the citizens of the community within which it operates. Every citizen has the right to food, health, shelter, justice, safety. Public services are thus aimed at improving the conditions of citizens, by granting a decent level in all the fundamental aspects of living.

A way of phrasing the PA mission is by saying that, whenever a citizen's right fails to be actualised, a citizen's need arises, and the satisfaction of that need is what should drive the PA in the first place.

Nonetheless, citizens' fruition of rights is not the only purpose for the implementation of public services, as well as the violation of fundamental rights is not the only trigger of citizen's needs. Citizens have life plans, goals, desires that drive their actions and their interactions within a society. In many cases, to pursue their own private goals within a society, people have to interact with the PA. For instance, when a citizen wants to buy a car to freely circulate, they have to interact with the authority for motor vehicles in order to satisfy their goal. That is, one needs a driving licence to circulate. Hence, a second view of need emerges here, not arising from violations or rights, rather emerging from the goals and the viable means to satisfy those goals ${ }^{1}$. Accordingly, a demand for

\footnotetext{
${ }^{1}$ An alternative way to present this example is to say that holding a driving license is a requirement to circulate in public streets. It is nonetheless a state that has to be reached in order to be able to execute the action
} 
public services arises, requiring the PA to respond, hence public services may also be motivated by citizens' need to satisfy their goals.

We are here embracing a view of public services that can be termed need-driven. To properly articulate such a view, a thorough analysis of needs is in fact required.

Even before disentangling the heterogeneous notions of need at work, we have to separate self-ascribed and hetero-ascribed (e.g. from the PA) needs [1]. In the former case, a citizen believes to need something for which an action of the PA is required. Citizens can be entitled to request to the PA to enact a service and the PA may respond to such request; we will call this kind of services reactive. In the latter case, it is the PA that attributes a need to a citizen, usually by proposing a service to satisfy it; we will call these proactive services. Proactive services are considered particularly desirable as they reduce the charge on the side of the citizens and allow the PA to fully achieve its mission; on the other hand, they require the PA to be able to correctly ascribe needs to citizens.

In principle, citizens know better their own needs, as they have first person experience of what they miss, while the PA knows better which are its means to cope with needs. However, this is not always the case, for various reasons. First of all, some citizen (e.g. cognitively impaired citizens or unaccompanied minors) could be not aware - while the PA is - of being in need. Secondly, citizens may know to be in need, but they do not know what to demand to the PA, because they are not aware of the services the PA may offer. Also, sometimes the state of need is associated with pain, is pressing, and prevents people from taking prompt action (e.g. health conditions). Thus, in some situations proactive services are required. Moreover, there is a series of needs that are easily predictable for the PA, either because they emerge from the demands of the PA itself (e.g. the issuance of a driver license to allow citizens to drive) or because they are associated with the occurrence of regular life-events (as the birth of a child).

Correctly ascribing needs to citizens is the first step to implement useful proactive (or even predictive) services. ${ }^{2}$ The second and fundamental step is to design services for each particular kind of citizen (youngsters, families, elderly, people with disabilities, foreigners, citizens belonging to minorities etc.) to help them satisfy their specific needs. The final phases are the delivery of services (specific deliveries to specific persons) and their ex-post evaluation. When designing a service system, all these steps are important.

While most of these phases require massive data gathering, the design phase, whose importance is well acknowledged and explained (for instance in [3]), presupposes a thorough analysis in (at least) two directions. The first one is prominently sociological and is dedicated to understand what people in certain conditions need. The other is conceptual and focuses on how the information required by the system can be organised and represented in a structured and well-founded manner, to promote accessibility and interoperability. This paper provides a contribution to the conceptual analysis at the design phase, by concentrating on the notion of need. When one talks about need-driven services, at least three different concepts are connected with the use of the term "need", both in com-

of circulating in a public street. On the other hand, it is in virtue of the final goal of circulating on public streets that citizens aim at holding a driving license, that is thus instrumental with respect to the end goal. We thank an anonymous reviewer for the suggestion.

${ }^{2} \mathrm{~A}$ fairly intuitive definition of proactive vs. predictive services is provided in [2], where the former are the services connected to needs whose emergence can be foreseen, but without an exact timing, while the latter are those triggered by needs for which the PA can foresee not only that they will emerge, but also when. 
mon sense and in scientific discourse [4]. Three very common uses of the term that can be found in the literature are listed below:

1. an event or a state that, if realised, brings about an end goal for an agent (these are referred to in some literature as "satisfiers" - see, e.g., [5] and [6]), for instance the need for a train, bus, or shuttle service to connect the airport with the city center;

2. an event or a state that is, besides sufficient, necessary to realise for the achievement of an end goal, i.e. if not realised, it prevents the end goal from being achieved (in the philosophical debate these have been defined "instrumental needs" [7], i.e. needs whose satisfaction is instrumental to the achievement of some end goal), as for example the need of having a driver license issued for being allowed to circulate on public streets with a car;

3. a very fundamental condition, whose absence causes an important damage for persons, universalisable goals [8] (these are sometimes called "absolute" or "basic" needs, sometimes "pre-conditions"). Examples are the need for food or freedom.

Need-driven services are therefore conjugated differently, according to the notion of need they refer to. In the first case, satisfying needs would mean finding solutions to help citizens to realise their own goals. In the second case, satisfying needs serves to provide the citizens with those means without which they could not achieve some of their goals. In the third case, copying with needs would translate into prioritizing the intervention on the most basic and important aspects of their citizens' lives. For sure all these aspects are desirable and should be included in the implementation of need-driven services, but a conceptual clarification is mandatory to be able to reason on such complex scenarios.

The objective of this paper is thus to provide a clear map of the various notions of need, by proposing a conceptual analysis as well as a formal ontology of needs. As suggested by the previous discussion, there is a connection between goals, as well as other mental attitudes, and needs. For this reason, we shall confront our treatment with the BDI (Belief-Desire-Intention) approach to mental attitudes, which provides wellestablished models and tools in knowledge representation. However, as we will see, to cope with the complexity of the notion of need, we shall complement the BDI view with the rich tradition of intentionalty in philosophy of mind. To provide a formalisation of our approach, we shall place our treatment within the foundational ontology DOLCE, as it is expressive enough for our purposes. The remainder of this paper is organised as follows. Section 2 approaches mental states on the basis of the philosophical view of intentionality, relates this view to BDI, and discusses this view wrt. the assumptions of DOLCE. Section 3 focuses then on needs, by discussing them as mental states and articulating the intentional nature of needs. Section 4 proposes our formal ontology of needs. Section 5 illustrates our view by means of an example of needs in public services. Section 6 concludes.

\section{Mental states and intentionality}

In this paper, we locate needs, from an ontological point of view, among those mental states that exhibit the feature of intentionality, namely the property of being about some- 
thing, i.e. about intentional objects. Although there are different types of mental states (e.g. beliefs, desires, expectations, perceptions etc. and also needs), they all share the property of intentionality, which somehow links them to their intentional object (e.g. if Maria loves Luigi, then "Luigi" is the intentional object of Maria's intentional state of love). In contemporary philosophical literature, reams of paper have been written on the notion of "intentional object"; nevertheless, there is currently no consensus about the ontological status of such objects. In fact, mental states can be about very different kinds of entities, for example, ordinary objects, properties or qualities, events, states of affairs. Besides, even nonexistent and impossible objects might be mental states' intentional objects (in literature, classic examples are Zeus, Pegasus, the golden mountain, the round square, etc.). Anyway, before taking into consideration intentional objects, we have to introduce some philosophical positions that we endorse in this paper regarding intentionality.

\subsection{Aboutness as directedness or reference}

According to a traditional picture of intentionality, which stretches back to Twardowski, this relation can be subdivided into two types of intentional binary properties that can be instantiated by mental states, namely having a content (directedness or aboutness $s_{1}$ ) and being about an object (reference or aboutness 2 ). As Haldane suggests [9, pp. 17-18], the difference between directedness and reference is that the former puts a mental state in connection with an intentional content, an entity whose kind is typically classified as abstract, the latter links a mental state with an intentional object. It is worth just briefly outlining that the intentionality of a mental state is independent of the existence of its objects, i.e. we can think about existent objects as well as nonexistent ones. How shall we explain this fact?

To answer this question, we should understand how directedness and reference interact. Regardless of the fact that the objects of mental states exist, we are able to cognitively grasp something, namely a contentful meaning. Directedness is equivalent to contentfulness and pertains to every mental state. Having an intentional content is a feature which is independent of the existence of objects because, at least in some cases, there are no objects which we can refer to, but still our mental states have a content. Thus, if Maria fears Boogymen, then Maria's mental state is related by means of directedness to the intentional content expressed by the term "Boogymen". Instead, when mental states' objects exist, directedndess has the "power", so to speak, to identify a specific object, an intentional object, which we can refer to. For example, imagine that Paul admires Varenne, the best trotter of all time. In this case, it is in virtue of directedness that his mental state is related to an intentional object through a reference relationship, because Varenne, among all existent entities, is the one which satisfies the intentional concept expressed by the definite description "the best trotter of all time". So, it is in virtue of having a content that mental states can refer to objects, provided that such objects exist. By now it should have become clear that the intentional picture sketched above is the counterpart, at the level of philosophy of mind, of Frege's notion of sense $\mathrm{e}^{3}$ : the sense of an expression is, in the Fregean view, what enables to determine its reference.

\footnotetext{
${ }^{3}$ Crane stressed [10, p. 21] that there is a complex relation between intentionality and intensionality, namely, there are linguistic contexts in which sentences do not satisfy certain extensional criteria.
} 


\subsection{Intentional objects as roles}

Now let's turn our attention to intentional objects. As already mentioned at the beginning of this section, the locution "intentional object" can be used in different senses. Trivially, every kind of entity can be an intentional object inasmuch as an entity is thought of. This leads Crane to affirm that an intentional object is not a particular kind of entity among others since, as long as something is the "target" of a mental state, every kind of entity counts as an intentional object. In other words, intentional objects are not a genuine category to be included in the ontological inventory, in Crane's words "they have no nature of their own" [9, p. 16]. We agree with Crane that our mental states can be about (in terms of reference) different types of entities and, in our account, we treat them as roles.

The notion of "role" has been discussed from several perspectives in quite different disciplines, from sociology to philosophy, as well as knowledge engineering and formal ontology ${ }^{4}$. We take here the classical approach for analyzing roles based on the works of Sowa [17,18], and Guarino [19]. In short, Sowa claims that a role is a monadic property which can be predicated of different entities; in technical terms, different entities can play the same role. For example, the role "customer" can be played by a person as well as by a company. Furthermore, an entity plays a role only with respect to a "pattern of relationships". For instance, the role "university student" holds only within a binary relation of "enrollment" to a university. Guarino adds constraints to Sowa's theory affirming that roles must be founded and anti-rigid. The notion of foundation or, better, generic foundation, is a binary relation between species (kinds of objects). It has been formulated by Husserl and expresses the idea that a property ${ }^{5} \alpha$ cannot exist as such except in a more comprehensive unity whit a property $\beta^{6}$. For example, a university student is such as founded on a university, and a wife is such as founded on a husband and vice versa. The former is a one-sided foundation relation (not symmetric), the latter is a two-sided or mutual foundation (symmetric) [20, p. 128]. Simons defines [9, p. 125] generic foundation as follows: property $\alpha$ is founded on property $\beta$ if and only if any instance $x$ of $\alpha$ is necessarily associated with an instance $y$ of $\beta$ which is not related to $x$ by a part-of relation.

Thus roles are founded properties, for example, "supplier" is founded on "customer" and vice versa (besides, the same person could play different roles simultaneously or at different times). It is worth noting that if an entity $y$ instantiates the property $\beta=$ person and the property $\alpha=$ student, then $y$ can cease to be a student (or whatever role you

\footnotetext{
${ }^{4}$ Some important works on roles are: [11], [12], [13],[14]. In 2007 the journal Applied Ontology published a whole special issue, in which the notion of role has been analyzed under an interdisciplinary perspective: [15]. A more recent approach on roles has been proposed by Mizoguchi and colleagues [16].

${ }^{5}$ Simon and Correia use the terms "kind" or "species", but we rather preferred to use the more generic term "property", in order to prevent confusion with how such terms are used in the literature on knowledge representation.

"The notion of "foundation" is used by Husserl to characterise the concept of "pregnant whole", that is a whole in which each part is "foundationally connected, directly or indirectly, with every other, and no part of the whole so formed is founded on anything else outside the whole" [20, p. 122]. Husserl interpreted the foundation relation as a necessary association between kinds. The father of phenomenology conceptualized a pregnant whole as a more comprehensive union between kinds, differently from a mereological aggregate. Simons has stressed [20, pp. 122-125] that if we formulated the connection between instances of different kinds in terms of parthood or proper parthood, we would not be able to capture Husserl's original intuition about pregnant whole and foundation.
} 
like) without changing its identity conditions, but can't cease to be a person, otherwise $y$ would be a different entity. In other words, "being a person" is a rigid property, that is in every possible world this property applies to all its instances necessarily. Instead, "being a student" is an anti-rigid property, namely, it doesn't apply necessarily to all its instances.

This being said, let us come back to the analysis of intentional objects. What is it to be an intentional object? With respect to a certain entity, we suggest that being an intentional object is nothing but playing a certain role within an intentional relationship, that is aboutness 2 .

We believe that aboutness 2 is a one-sided relation holding between an agent's mental state ' $\mathrm{X}$ ' and an entity ' $\mathrm{Y}$ '. Now, the point is that the role of intentional object $z$ is played by $y$ to the extent that $x$ is about $2 y$. For instance, suppose that Paul admires Varenne. Given that Varenne is related to Paul's mental state by means of aboutness 2 , Varenne as such plays the role of intentional object. It is worth noting that we are not embracing here an ontological multiplicative approach, that is we are not stating that the intentional object Varenne is a different entity from Varenne. All we are saying is that only insofar Varenne is the "target" of Paul's thought it instantiates a certain quality or property, which is the property of being an intentional object. We believe that two interesting consequences can be deduced from these observations.

First of all, let the property $\Phi$ stand for the property "being an intentional object" and $\Psi$ for the property "being a mental state". We can affirm that any instance $x$ of $\Phi$ is necessarily associated with an instance $y$ of $\Psi$ which is not related to $x$ by a part-of relation (where the association relationship between instances corresponds to aboutness 2 ). So $\Phi$ is founded on $\Psi$. In addition, the property $\Phi$ is anti-rigid. In fact, it is only to the extent to which an entity is involved in an intentional relation (aboutness $s_{2}$ ) with a particular mental state that this entity instantiates the property $\Phi$. Since $\Phi$ is a founded and anti-rigid property, $\Phi$ is a role. To sum up, $\Phi$ is a monadic property that can be predicated of different entities and this is a welcome result if one wants to maintain the idea that ontologically different kinds of objects can be intentional objects ${ }^{7}$.

\subsection{Intentional contents and social concepts in DOLCE}

It is time to make some clarifications on the approach we are developing on intentionality, adapting the traditional philosophical picture introduced above to DOLCE, that is the top-level ontology on which we decided to ground this work. The choice of DOLCE is motivated by its ontological commitment to its being tailored to common-sense representations of cognitive agents, rather than on the constitution of the "reality" as prescribed

\footnotetext{
${ }^{7}$ Sometimes individuating entities that play the role of being an intentional object could be a tricky matter. For instance, if Maria believes that Naples is the largest city of Southern Italy, one may claim that the intentional object wrt. Maria's mental state is the state of affairs "Naples is the largest city of Southern Italy". But others could argue that, actually, we can individuate two different intentional objects, that is "Naples" and the property "being the largest city of Southern Italy". In fact, given that a state of affairs is made up of its constituents, holding this thesis would be equivalent to saying that Maria's intentional state of belief is about (in terms of reference or aboutness 2 ) the property "being the largest city of Southern Italy" instantiated by "Naples", namely the state of affairs "Naples is the largest city of Southern Italy". Hence we suggest that it would be more accurate to say that Maria's intentional state is derivatively about the state of affairs qua complex entity. See [21, p. 29].
} 
by science. This feature brings to the core of DOLCE the importance of modelling the mental and the social realms.

With this in mind, we know that intentionality is a relation that can be subdivided into two types of intentional binary relations, namely having a content (directedness or aboutness ${ }_{1}$ ) and being about an object (reference or aboutness 2 ). Concerning the first relation, we said that the intentional content is a contentful meaning, something that we are able to grasp cognitively, and that a mental state can succeed in individuating intentional objects (in terms of reference) in virtue of its having an intentional content. Ontologically speaking, we interpret intentional content with the notion of Social Concept ${ }^{8}$. Social concepts are described in $[22]^{9}$ as particulars that are created and accepted by a community of intentional agents and defined by descriptions encoded in linguistic expressions. Concepts can classify different kinds of entities, for example, objects as well as events ${ }^{10}$. Since our aim in this paper is representing the social domain of public services that, similarly as in [23], we see as events, we will specifically take into consideration concepts that classify events.

For example, in some countries parents are automatically entitled to receive a child benefit when a child is born. "Providing a child benefit" is a proactive service that can be seen as a concept whose description could be recorded in a PA's official document. Each service provision counts as a single event that is classified by the concept defined by the description. Concepts classify entities that satisfy all the constraints in the concept definition. Furthermore, the classification relation is associated with a time parameter that identifies a specific interval in which the classified entity satisfies the definition, more formally $\operatorname{cf}(x, y, t)$ stands for "the social concept $x$ classifies the event $y$ at time $t$ ". In the example, if the social concept is "Providing a child benefit", all the events that satisfy the constraints set by it at a certain time are the events classified by it at that time, for example the event "Giovanni sends the payment on Lucia's bank account" at 3.52 PM on April 14, 2020 satisfies such constraints and is thus classified as a social concept of "Providing child benefit".

This being said, from an intentional standpoint, when a citizen needs that the PA provides them with a child benefit, their mental state of need will be directed (directedness) towards the concept "PA providing a child benefit". Furthermore, in virtue of this concept, the citizen's mental state will refer to (reference) an intentional object that is one among a set of events that are classified by that concept (provided there are any) .

It is worth noting that two different concepts can classify the same entity, for instance, the event "Giovanni sends the payment on Lucia's bank account" could be classified by two different concepts, for example "PA providing a child benefit" if certain conditions hold, like that Giovanni works in a PA and that payment is connected to child benefits etc. and "paying off a debt to a friend" if other conditions hold (at different times

\footnotetext{
${ }^{8}$ Following DOLCE, in this paper we endorse a social view of concepts, i.e. we see them as strictly connected to (natural) language and intersubjective. We do not take them to be "private" entities existing only in the mind of one agent. That concepts are dependent on the social nature and practices of language is very well established in the philosophical literature, and we are here endorsing this position, without entering in the debate on whether private concepts exist.

${ }^{9} \mathrm{~A}$ similar account was previously presented in [13] for social roles, defined as social concepts that classify enduring entities, like objects.

${ }^{10}$ With the term "event" we are referring here generally to perduring entities, including states, processes and events in a stricter sense. From now on we will use "event" in this wide sense.
} 
or simultaneously). The same event can then be grasped in different ways, similarly as it happens with concepts in many theories of intentionality.

This highlights that concepts can be considered good candidates for being intentional contents. Another reason is that concepts are somehow "shareable" between different agents. Since descriptions, and therefore social concepts, are accepted by a community of intentional agents, different agents can cognitively grasp the same concept and hence refer to the same intentional object. Furthermore, it is worth noticing that, analogously as for intentional contents that fail to refer to nonexistent objects (like Pegasus), also social concepts can exist without classifying any entity (at a certain time); as an example, we can talk about the king of Italy, though at the moment there is no entity classified by such concept. The current discussion is depicted in Figure 1.

Lastly, intentional contents can be seen as roles just like intentional objects. In fact, "Being an intentional content" seems to be a founded and anti-rigid property. Briefly, we note that: A) any instance of the property "being an intentional content" is necessarily associated with an instance of the property "being a mental state" without involving a partof relation between instances and through an association relationship that corresponds to the intentional relationship of directedness. Thus the former property is founded on the latter. B) If we see concepts as entities that play the role of intentional content, only as long as a concept is involved in a directedness relationship with a particular mental state this concept instantiates the property of being an intentional content. Hence, being an intentional content is an anti-rigid property.

To sum up, we have characterised mental states in DOLCE as being about (directed $^{\text {(ding }}$ to) social concepts and being about 2 one of the entities that are classified by those concepts. In the next section we will see how this analysis can be specified to account for needs.

aboutness 1

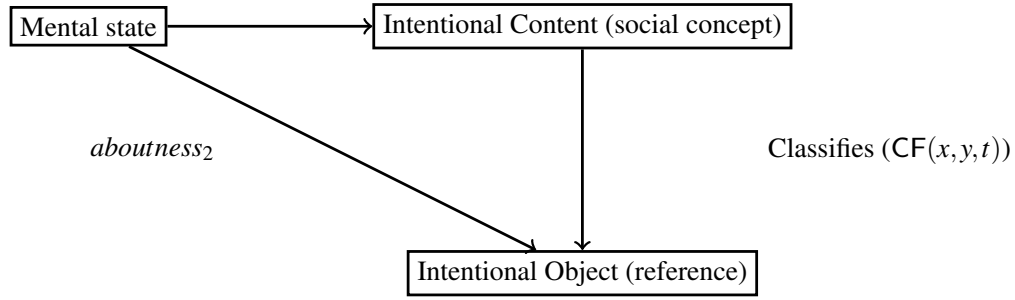

Figure 1. Aboutness relations

\section{Needs as mental attitudes}

As anticipated, to represent the notion of need, we start from the perspective on mental attitudes of BDI, because it is based on a long established tradition in knowledge repre- 
sentation $^{11}$, and it has been implemented in many widely used applications (e.g. JAM, Jason, and SPARK ${ }^{12}$ ).

Most approaches based on BDI have been developed for reasoning and planning with mental attitudes but, as far as we know, not much effort has been spent to define what they are and what they are about from an ontological point of view ${ }^{13}$. There seems to be a tension between the fact that mental attitudes are traditionally conceived of as being about propositions (they are also often identified with "propositional attitudes"), and the fact that a real planning agent, while realising a proposition, brings about changes in the real world. Moreover, the fact that mental attitudes are about something is implicit in the BDI representation framework. For this reason, the intentionality-based view of mental attitudes nicely complements the BDI view.

In classic BDI approaches, beliefs, desires and intentions are seen as the fundamental mental entities dependent on an intentional agent, with different features (which in the philosophical debates have been called "intentional modes" [31], [10]): while beliefs are informational - they constitute the information available to the agent - , desires are motivational - they motivate the agent to act - and intentions are deliberative - given bounded rationality, they allow the agent to commit to a course of action, until it is fulfilled or it fails.

Turning now to needs, in light of our discussion of intentionality, we can ask a number of questions: which kind of mental states are needs? What is their intentional content? What is their intentional object(s)? A first assumption, which is quite well established in the literature, is that needs are mental states separated from desires, beliefs, and intentions.

Let's now recall the different uses of the term "need" listed in the Introduction:

1. an event that, if realised, brings about an end goal for an agent (the need of taking a train/bus/shuttle to go to the airport);

2. an event that, if not realised, prevents the end goal from being achieved (the need of having a driver license issued to circulate in public streets);

3. a very fundamental condition, whose absence harms the person (the need for food $)^{14}$.

A first thing to notice, these are not definitions of what is a need, but of what is needed, of what such needs are about prima facie. In other terms, these correspond of what in the literature are called "satisfiers". As we shall see, some of the features of the satisfiers identify different concepts of need.

In [7], needs are construed as goals, where a goal is intended as a chosen desire, i.e. a desire that has been selected at a certain time among possibly conflicting desires, as the one to be pursued. Goals can be subdivided in end goals and instrumental goals; end goals are goals that are pursued per se, while instrumental goals are goals whose satisfier

\footnotetext{
${ }^{11}$ Seminal contributions are [24], [25] and [26].

${ }^{12}$ See http://www.marcush.net/IRS/, http://jason. sourceforge.net/wp/, and http://www . ai.sri.com/ spark/

${ }^{13}$ There are also approaches to BDI using BFO (Basic Formal Ontology), e.g. [27] and [28], and UFO (Unified Foundational Ontology), e.g. [29] and [30]

${ }^{14}$ One could object that in all these cases, the need is about an endurant, rather than a perdurant, for instance the need of a train, of a driving license or of food. But our intuition is that these are just elliptical ways to express needs of some events to happen. For example, someone does not just need a train to exist, they need to be able to take it.
} 
- in this case an event -, if realised, brings about the satisfaction of the end goal (makes the event which the end goal is about occur).

If we look back at the three uses, the first two seem to refer to satisfiers of instrumental goals, while the latter of an end goal. But let's analyse them in light of the previous discussion on intentionality.

The first thing to be noticed is that all three refer to an event having certain properties. By viewing needs as mental attitudes, as we argued, they have: $i$ ) as a content a concept (i.e they are mental states about ${ }_{1}$ a concept) that classifies events with certain characteristics; $i i)$ as a reference (about ${ }_{2}$ ) one among the classified events (the satisfier).

Going further, let's call need ${ }_{1}$ and need ${ }_{2}$ the mental attitudes associated with uses 1. and 2. respectively; they are apt to be represented by instrumental goals. But what distinguishes one from the other? Our proposal is to distinguish them depending on the relation that their satisfiers bear with the events that are the intentional object of the connected end goal. Instrumental goals have satisfiers that, if realised, can bring about an event that is the intentional object of the end goal.

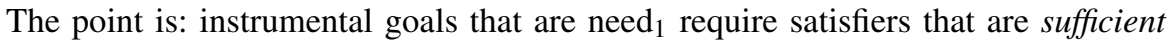

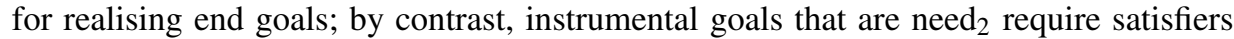
that are, besides sufficient, also necessary for realising end goals. This seems to capture our common sense distinction between saying "I need to do $x, y$ or $z$ to obtain $w$ " (" $x, y$ or $z$ could be of help") and "I need to do $x$ to obtain w", the latter conveying a stronger message and an implicature ("I cannot do without it").

Point 3. seems instead to be connected to a different kind of need, what has been defined by some scholars an "absolute need". We thus introduce need ${ }_{3}$ to talk about those needs whose satisfiers are states, conditions which are aimed at independently of any further goal and whose absence causes in the agent a consequent mental state (a state of need).

These three notions of need all play an important role for the PA: need ${ }_{1}$ can be used to identify the different services that the PA may offer to help citizens to achieve their goal; need ${ }_{2}$ can be used to single out those services that should be necessarily provided to citizens when the PA "legitimates" their goals on an institutional level and thus assumes them; need ${ }_{3}$ can be used to drive the priorities of the PA towards goals which, if not reached, can compromise the well-being of the citizens.

\section{A DOLCE-based ontology of intentional mental attitudes and needs}

We develop our formal approach within DOLCE, so the following axioms are designed to be added to DOLCE, cf. [32] ${ }^{15}$. Mental states (MS) are a type of states (ST, cf. [32], p. 24), i.e. a type of perdurants (aka events, which are disjoint from endurants, from time intervals, and from concepts) ${ }^{16}$, cf. axiom (a1) ${ }^{17}$. Participation $\mathrm{pc}(x, y, z)$ (see [32], p.

\footnotetext{
${ }^{15}$ We implemented our ontology and we tested its consistency as well as its provable consequences, cf. https://github.com/diporello/DOLCE-mental_states-needs/blob/master/dolce_needs.p for the details. This treatment is also compatible with [33]. We present an excerpt of the axioms here.

${ }^{16}$ This is the view of concepts in DOLCE-CORE, cf. [22].

${ }^{17}$ States in DOLCE are perdurants that are cumulative and homeomeric. E.g. "sitting", the sum of two instances of sitting can still be a sitting and the parts of sitting are all sitting states. By viewing mental states as states (and not processes), we abstract here from the internal articulation of mental states. Moreover, here states are perdurants, so states happening at different times are different. A contrasting view of states is in [34].
} 
20 ) is a ternary relation connecting an entity, a perdurant (i.e. also a state), and a time interval (T, cf. [32], p. 74). Existence is represented by the binary relation "present at", pre $(x, t)$ (cf. [32], p. 37). Axiom (a2) states that every mental state depends on an agent who participates to that state (APO, agentive physical object, cf. [32], p. 24). Moreover, a mental state is private to the agent: for all time intervals, the same agent participates to the mental state, cf. (a3).

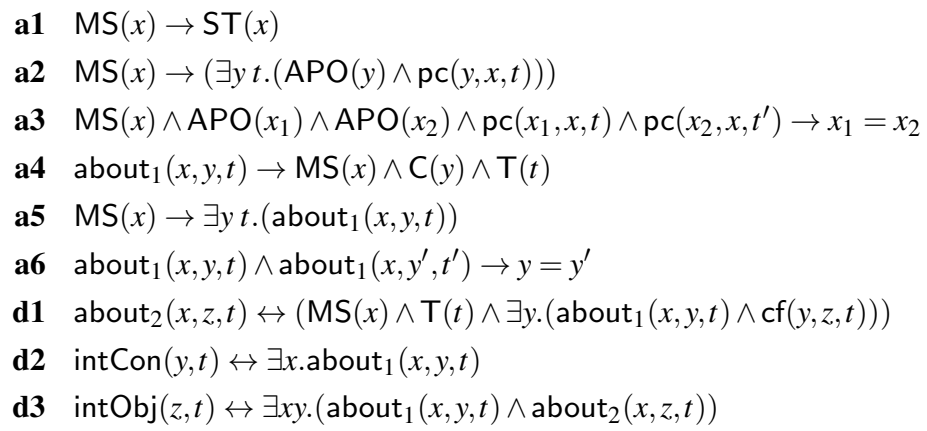

The intentional content of mental states is treated in (a4)-(a6): about ${ }_{1}$ relates mental states and concepts, as we discussed in Section 2, cf. (a4). Every mental state must be about $_{1}$ a concept, cf. (a5), and by (a6) a mental state is associated to exactly one concept. Hence, two mental states that are about different contents must be different. ${ }^{18}$

The intentional object of mental states is expressed by the relation about 2 , cf. (d1). According to (d1), the reference of a mental state can be undetermined, if more entities are classified by the concept, or even empty, if the concept does not classify anything at a time. Also, the reference may change through time, depending on which entities are classified by the content of the mental state at that time. ${ }^{19}$

As we discussed in Section 2, intentional objects and intentional contents are roles played, respectively, by concepts and general entities, cf. (d2) and (d3).

We start the treatment of the different notions of need and of the relationship between needs and goals, by introducing the view of needs as mental states. By specialising the type of mental states, we can represent beliefs, desires, intentions, as well as needs, as mental states, i.e. $\mathrm{BS}(x), \mathrm{DS}(x), \mathrm{IS}(x), \mathrm{NS}(x)$ are disjoint subtypes of MS. This view assumes, for instance, that a state of need differs from a state of desire. ${ }^{20}$

To reason about mental states (à la $\mathrm{BDI}$ ), we introduce the relations bel $(i, y, t)$, $\operatorname{des}(i, y, t)$ and $\operatorname{int}(i, y, t)$, whose meaning is " $i$ believes, desires, intends $y$ at $t$ ". These relations are defined by constraints such as (d4). ${ }^{21}$ Accordingly, to express the content

\footnotetext{
${ }^{18}$ The motivation for (a6) is that we do not want to enter the complex issue of the internal structure of a mental state and its relation with the structure of its content. Whether the internal structure of a mental state resembles the logical structure of its content is a very challenging open question of cognitive science.

${ }^{19}$ Concepts in DOLCE must be instantiated in at least one possible world. This excludes impossible concepts, e.g. the round square. To enable impossible concepts as content of mental states, we need to abandon that constraint of DOLCE.

${ }^{20} \mathrm{We}$ are admittedly vague in not endorsing a specific view of mental states. For instance, we do not want to enter the debate on the relation between mental states and neural (physical) states. The motivation is to propose an ontology of need that is hospitable to different views of mental states. Also, we do not enter the debate on what separates a need state from a desire state, viewing this problem as a direct question for cognitive science.

${ }^{21}$ Since concepts may classify events and since events are in the domain of DOLCE, assuming that the content of a mental attitude is a concept is not restrictive wrt. assuming that the content is a proposition, as in BDI. A
} 
and the agent of a need state, we use the relation termed need0, cf. (d5): $i$ needs0 $y$ at time $t$ if and only if there exists a need state $x$ to which $i$ participates that is about $y$. The relation need 0 expresses that a certain agent is in a mental state of need about $y$ at a time $t$.

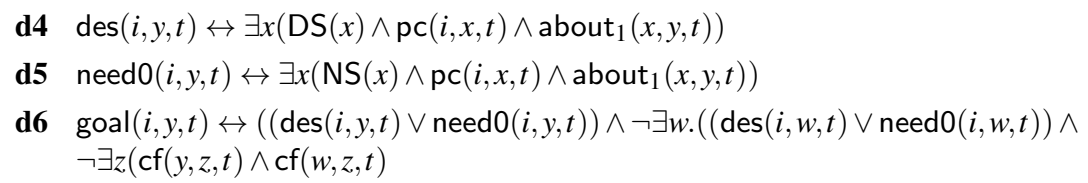

Goals are defined as desires or needs of an agent that are not incompatible with other desires or needs (thus they can be chosen). We express goal attitudes by the relation goal $(i, y, t)$ and we define them in (d6): agent $i$ has goal $y$ at $t$ iff $i$ desires or needs $y$ at $t$ and $i$ has no desires or needs incompatible with $y$ (where desires and needs are incompatible if they are about concepts that cannot be simultaneously satisfied). Also notice that Definition (d6) assumes that goals can be associated to a state of desire as well as to a state of need.

We separate instrumental goals igoals $(x, y, t)$ and end goals egoals $(x, y, t)$. To define these types of attitudes, we firstly introduce the notion of satisfier of a goal, (d7), i.e. an (existing) event that satisfies the content of the goal (need). ${ }^{22}$ Secondly, we suppose that time intervals can be strictly ordered by $\prec$. Finally, instrumental goals are those that, if satisfied, bring about an event that satisfies a subsequent goal (the end of the instrumental goal), cf. (a8). By contrast, end goals do not need a further goal to be satisfied, in this sense, they are pursued per se. ${ }^{23}$

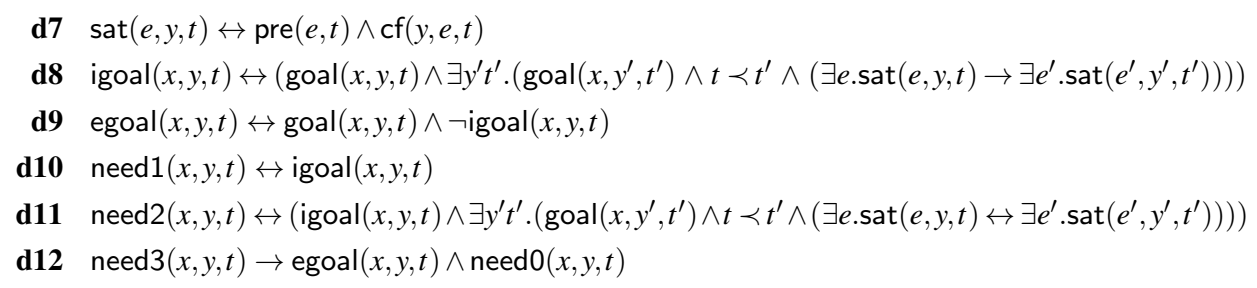

We can now introduce our refined notions of need, besides needs as mental states (need0): need1 views needs as instrumental goals cf. (d10), whereas need2 views needs as goals whose satisfiers are both necessary and sufficient for the end goal, cf. (d11). Accordingly, need 2 entails need1 (i.e. igoal). Finally, (d12) introduces the view of needs as end goals which are associated to a need state, corresponding to the absolute view of needs; accordingly, need3 implies need0. By contrast, neither need 1 nor need 2 implies need0: need1 and need2 are both igoal, which might be associated to desire, and not to need, states.

proposition classifies states of affairs as here concepts classify states or events. A similar approach, that views the content of mental attitudes as types, is proposed in [35].

${ }^{22}$ DOLCE views the elements of the domain as possibilia (by adopting the QS5 modal axioms), so the domain is intended to include possible events.

${ }^{23}$ To enable goals that have future satisfiers (e.g. at $t_{0}$ my goal is to eat supper at $t_{1}$ ), we can enable temporal indexes in the definition of the concepts, expanding on the formalisation of concepts in [13]. We leave this aspect for a future work. 
To sum up, we formalised the four uses of the term "need" that we discuss in this paper: $i$ ) needs as mental states of agents (cf. NS(x), whose content and agent can be expressed by (d5)); ii) needs as instrumental goals, (cf. need1 and (d10).); iii) needs as necessary instrumental goals (need2 and (d11).); $i v$ ) needs as end goals associated to a state of need (need3 and (d12).)

\section{A guiding example}

From the just presented perspective, the PA services offering to citizens can be seen as a manner to provide satisfiers to needs. When the PA ascribes a need to a citizen, the content of such need is a social concept classifying events with certain features. At execution time, the PA brings about an event (with actual individual agents, specific time, place etc.) so that the executed service (hopefully) ends up being a satisfier of the need. In the ex-post evaluation, the PA will check whether the specific instance of service that has been executed possesses in fact all the properties that characterise the concept of the service and if the final outcome of the execution of the service is in fact the achievement of the citizens' goal.

Let's see now with an example why all the different notions of needs that have been formalised are useful when representing public services, in particular proactive services, activated in conjunction with a "life event" generating new needs for citizens (like having a child, looking for a new job, starting education...).

Suppose that Maria is a low income single woman who just had a baby. Very likely, such event puts Maria in a number of states of need NS(x), whose contents can be specified by our relation need0. Allegedly, the life event "having a child" will generate a series of new goals for Maria. A first goal will likely be an end goal, namely that this child enjoys a state of well-being, so that they have food, clothes, diapers, etc. Having enough money to be able to make all the required expenses is for sure an instrumental goal with respect to Maria's end goal. But, by analysing Maria's income and her economic situation in general, the PA may understand that there is an only way to grant Maria the possibility of affording all the necessary expenses, and that is to provide Maria with some more income. So, Maria's need to receive some additional income will trigger a single parent's child allowance, one of the services that the PA offers. Since providing a single parent's child allowance is a necessary instrumental goal w.r.t Maria's end goal, this can be represented by need 2 .

A different PA could, on the other hand, provide in addition different services to meet the same needs, like for instance delivering food at home and temporarily lending clothes and diapers; in such case, all these services may satisfy the content of an instrumental goal, a need1.

Finally, in the example, Maria is not the only person acquiring a need with the birth's event: the child also acquires their "absolute" needs, like the need for food, personal autonomy, or freedom, and these are needs represented by need3.

\section{Concluding remarks}

This paper aims to contribute by building a dialogue between different areas of research: that of philosophy of intentionality, of BDI, and of formal ontology. With respect to the 
study of intentionality, the paper contributes, firstly, by characterising, with the help of ontological analysis, the notions of intentional object and intentional content as roles. Secondly, it leverages philosophy of intentionality to study the ontological nature of the intentional content and object of mental attitudes, identifying the former with social concepts and the latter with the entities that are classified by such concepts. Thirdly, it contributes to the studies on the notion of need, representing needs as mental attitudes and distinguishing different notions associated with the term "need". Fourthly, it extends the DOLCE ontology of mental entities, by integrating it with the treatment of intentionality and by extending it with the identified notions of need. Finally, it lays the bases for designing a service system in which services are triggered by various kinds of needs.

\section{References}

[1] L. Biccheri and R. Ferrario. An analysis of the notion of need for the representation of public services. In A. Barton, S. Seppälä, and D. Porello, editors, JOWO 2019 - The Joint Ontology Workshops, Proceedings of the Joint Ontology Workshops 2019, Episode 5: The Styrian Autumn of Ontology, Graz, Austria, September 23-25, 2019, volume 2518. CEUR Workshop Proceedings, 2019.

[2] H. Scholta, W. Mertens, A. Reeve, and M. Kowalkiewicz. From one-stop-shop to no-stopshop: An egovernment stage model. In I. Ramos, H. Krcmar, and V. Tuunainen, editors, Proceedings of the 25th European Conference on Information Systems, pages 918-934. Association for Information Systems, http://aisel.aisnet.org/ecis2017/, 2017.

[3] R. Sirendi, A. Mendoza, M. Barrier, K. Taveter, and L. Sterling. A conceptual framework for effective appropriation of proactive public e-services. In Proceedings of the European Conference on e-Government, ECEG, pages 213-221. Academic Conferences and Publishing International Limited, 2018.

[4] D. Gasper. Conceptualising human needs and wellbeing. In I. Gough and J. MacGregor, editors, Wellbeing in Developing Countries: New Approaches and Research Strategies, pages 47-70. Cambridge University Press, 2007.

[5] S. K. McLeod. Two philosophies of needs. Polish Journal of Philosophy, 9(1):33-50, 2015.

[6] M. Max-Neef. Human Scale Development. Apex Press, New York, 1991.

[7] C. Castelfranchi. To believe and to feel: The case of "needs". Technical Report FS-98-03, AAAI, 1998.

[8] L. Doyal and I. Gough. A theory of human need. Critical perspectives. Macmillan, 1991.

[9] J. Haldane. Brentano's problem. Grazer Philosophische Studien, 35:1-32, 1989.

[10] T. Crane. Elements of Mind: An Introduction to the Philosophy of Mind. Oxford: Oxford University Press, 2001.

[11] F. Steimann. On the representation of roles in object-oriented and conceptual modelling. Data and Knowledge Engineering, 35:83-106, 2000.

[12] F. Loebe. An Analysis of Roles. Toward Ontology-Based Modelling Master's Thesis. Master's thesis, University of Leipzig, 2003.

[13] C. Masolo, L. Vieu, E. Bottazzi, C. Catenacci, R. Ferrario, A. Gangemi, and N. Guarino. Social roles and their descriptions. In D. Dubois, C. Welty, and M. Williams, editors, Ninth International Conference on the Principles of Knowledge Representation and Reasoning, pages 267-277, Whistler Canada, 2004. AAAI Press.

[14] G. Boella and L. van der Torre. A foundational ontology of organizations and roles. In M. Baldoni and U. Endriss, editors, Declarative Agent Languages and Technologies IV. Springer, Berlin / Heidelberg, 2006.

[15] G. Boella, L. van der Torre, and H. Verhagen. Special issue: Roles, an interdisciplinary perspective. Applied Ontology, 2:81-207, 2007.

[16] R. Mizoguchi, A. Galton, Y. Kitamura, and K. Kozaki. Families of roles: A new theory of occurrentdependent roles. Applied Ontology, 10(3-4):367-399, 2015.

[17] J. F. Sowa. Using a lexicon of canonical graphs in a semantic interpreter. In M. W. Evens, editor, Relational models of the lexicon. Cambridge University Press, 1998.

[18] J. F. Sowa. Knowledge Representation: Logical, Philosophical, and Computational Foundations. Brooks/Cole, Pacific Grove, CA, 2000. 
[19] N. Guarino. Concepts, attributes and arbitrary relations: Some linguistic and ontological criteria for structuring knowledge bases. Data and Knowledge Engineering, 8(2):249-261, 1992.

[20] P. Simons. Three essays in formal ontology. In B. Smith, editor, Parts and Moments. Studies in Logic and Formal Ontology, pages 111-256. Philosophia Verlag, Munich, 1982.

[21] F. Dretske. Naturalizing the Mind. The MIT Press, Cambridge (MA), 1995.

[22] S. Borgo and C. Masolo. Foundational choices in dolce. In Theory and Applications of Ontology, volume 2, chapter 12. Springer Verlag, 2008.

[23] R. Ferrario and N. Guarino. Towards an ontological foundation for services science. In J. Domingue, D. Fensel, and P. Traverso, editors, Future Internet - FIS 2008, pages 152-169, Berlin, Heidelberg, 2009. Springer Berlin Heidelberg.

[24] M. Bratman. Intention, plans, and practical reason. Harvard University Press, Cambridge, MA, 1987.

[25] P. R. Cohen and H. J. Levesque. Intention = choice + commitment. In Proceedings of the Sixth National Conference on Artificial Intelligence - Volume 2, AAAI'87, pages 410-415. AAAI Press, 1987.

[26] A. S. Rao and M. P. Georgeff. Modeling rational agents within a BDI-architecture. In Principles of Knowledge Representation and Reasoning. Proceedings of the second International Conference, pages 473-484, San Mateo, 1991. Morgan Kaufmann.

[27] J. Hastings, W. Ceusters, M. Jensen, K. Mulligan, and B. Smith. Representing mental functioning: Ontologies for mental health and disease. In Towards an Ontology of Mental Functioning (ICBO Workshop), Proceeedings of the Third International Conference on Biomedical Ontology. 2012.

[28] A. Barton, W. Duncan, F. Toyoshima, and J. Ethier. First steps towards an ontology of belief. In L. Jansen, D. P. Radicioni, and D. Gromann, editors, Proceedings of the Joint Ontology Workshops 2018 Episode IV: The South African Spring co-located with the 10th International Conference on Formal Ontology in Information Systems (FOIS 2018), Cape Town, South Africa, September 17-18, 2018, volume 2205 of CEUR Workshop Proceedings. CEUR-WS.org, 2018.

[29] J. C. Nardi, R. de Almeida Falbo, J. P. A. Almeida, G. Guizzardi, L. F. Pires, M. van Sinderen, N. Guarino, and C. M. Fonseca. A commitment-based reference ontology for services. Inf. Syst., 54:263-288, 2015.

[30] D. Porello and G. Guizzardi. Towards an ontological modelling of preference relations. In C. Ghidini, B. Magnini, A. Passerini, and P. Traverso, editors, AI*IA 2018 - Advances in Artificial Intelligence - XVIIth International Conference of the Italian Association for Artificial Intelligence, Trento, Italy, November 20-23, 2018, Proceedings, volume 11298 of Lecture Notes in Computer Science, pages 152165. Springer, 2018.

[31] J. R. Searle. Intentionality: An Essay in the Philosophy of Mind. Cambridge University Press, 1983.

[32] C. Masolo, S. Borgo, A. Gangemi, N. Guarino, and A. Oltramari. Ontology library (wonder-web deliverable d18). URL: http://www. loa-cnr. it/Papers D, 18:36, 2003.

[33] R. Ferrario and A. Oltramari. Towards a computational ontology of mind. In A. C. Varzi and L. Vieu, editors, Formal Ontology in Information Systems, Proceedigs of the Intl. Conf. FOIS 2004, pages 287297. IOS Press, 2004.

[34] C. Masolo, A. B. Benevides, and D. Porello. The interplay between models and observations. Appl. Ontology, 13(1):41-71, 2018.

[35] D. Porello, G. Guizzardi, T. P. Sales, G. C. M. Amaral, and N. Guarino. An ontological account of the action theory of economic exchanges (full paper). In B. Roelens, W. Laurier, G. Poels, and H. Weigand, editors, Proceedings of 14th International Workshop on Value Modelling and Business Ontologies, Brussels, Belgium, January 16-17, 2020, volume 2574 of CEUR Workshop Proceedings, pages 157-169. CEUR-WS.org, 2020. 